\title{
Specific and label-free femtomolar biomarker detection with an electrostatically formed nanowire biosensor
}

\begin{abstract}
Gil Shalev ${ }^{1}$, Guy Landman ${ }^{1}$, Iddo Amit ${ }^{1}$, Yossi Rosenwaks ${ }^{1}$ and Ilan Levy ${ }^{2}$
We report a specific, label-free and real-time detection of femtomolar protein concentrations with a novel type of nanowirebased biosensor. The biosensor is based on an electrostatically formed nanowire, which is conceptually different from a conventional silicon nanowire in its confinement potential, charge carrier distribution, surface states, dopant distribution, moveable channel and geometrical structure. This new biosensor requires standard integrated-circuit processing with relaxed fabrication requirements. The biosensor is composed of an accumulation-type, planar transistor surrounded by four gates, a backgate, front gate and two lateral gates, and it operates in the all-around-depletion mode. Consequently, adjustment of the four gates defines the dimensions and location of the conducting channel. It is shown that lithographically shaped channels of $400 \mathrm{~nm}$ in width are reduced to effective widths of $25 \mathrm{~nm}$ upon lateral-gate biasing. Device operation is demonstrated for protein-specific binding, and it is found that sensitive detection signals are recorded once the channel width is comparable with the dimensions of the protein. The device performance is discussed and analyzed with the help of three-dimensional electrostatic simulations.
\end{abstract}

NPG Asia Materials (2013) 5, e41; doi:10.1038/am.2012.75; published online 1 march 2013

Keywords: biosensors; label-free; nanowire

\section{INTRODUCTION}

Biosensors are analytical devices that convert a biological response into an electrical signal. They are normally composed of two main components: the sensing device and the sensing molecule, that is, the chemical or biological recognition elements. As a functional hybrid system, the main challenge is to design a system that couples the unique features of the biorecognition event with sensitive signal detection and amplification. Many researchers have reported the potential utilization of nanostructure-based biosensors in the detection of biological species featuring direct electrical transduction.

Nanowire-based biosensors, in particular, are promising candidates for the transduction of analytical signals in solution into electrical signals, and ultrasensitive sensing has been demonstrated for ions, ${ }^{1}$ molecules, ${ }^{2}$ proteins, ${ }^{3-6} \mathrm{DNA}^{7}$ and viruses. ${ }^{8}$ Nanowires are fabricated either via bottom-up or top-down processes. However, bottom-up fabrication has thus far prevented the commercialization of such nanowire sensors mainly due its incompatibility with high-volume manufacturing. In contrast, various top-down methods entail a high level of non-standard processing complexity and high process variation. ${ }^{5,9-11}$ In addition, both methodologies produce three-dimensional nanowires that inherently suffer from a high density of surface states and non-uniform doping distributions that entail degradation in device performance. ${ }^{12-14}$ The development of nanofabrication tools and techniques, such as electron beam lithography, focused ion beam and nanoimprint lithography, has propelled the momentum of functional nanostructure-based biosensor fabrication. Altghough this rich inventory of nanofabrication methods has been available for many years, the methods still lack the capability for on-chip integration of nanostructure-based biosensors with an allied signal-processing circuitry and a fluid-handling module using standard microfabrication technology. A novel, double-gate nanowire field effect transistor for biosensing was recently reported. ${ }^{15}$ The device was patterned with $193 \mathrm{~nm}$ lithography, and specific sensing of $1.34 \mathrm{nmoll}^{-1}$ of anti-avian influenza was demonstrated.

In this work, we present an electrostatically formed nanowire (EFN) biosensor capable of specific, label-free and real-time detection of femtomolar protein concentrations. The EFN biosensor is composed of a nanowire-like conducting channel that is not physically fabricated, but rather, electrostatically formed post fabrication. Consequently, fabrication requires standard, integrated-circuit processes, thus eliminating the need of sophisticated nanoscale fabrication. Competitive performance coupled with standard silicon processing makes the EFN biosensor a viable technology for various real-time diagnostic applications.

${ }^{1}$ School of Electrical Engineering_-Physical Electronics, Tel Aviv University, Ramat-Aviv, Israel and 2Photocell, Hebrew University, High-Tech Village, Givat Ram Campus, Jerusalem, Israel

Correspondence: Professor Y Rosenwaks, Department of Electrical Engineering_Physical Electronics, Tel Aviv University, Tel Aviv 69978, Israel.

E-mail: yossir@eng.tau.ac.il

Received 10 April 2012; revised 29 October 2012; accepted 6 November 2012 
a

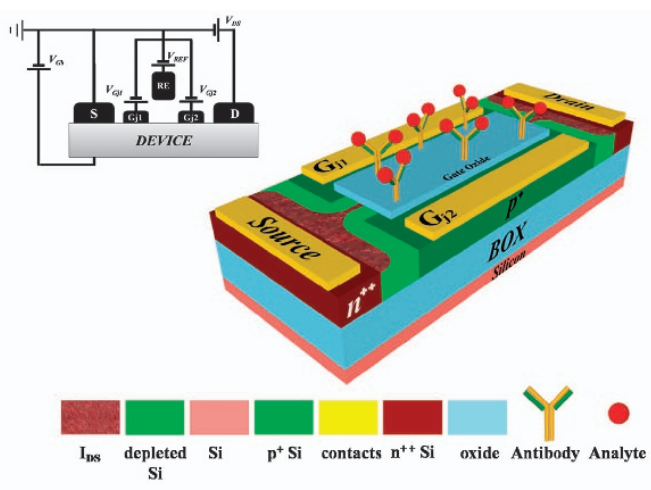

b

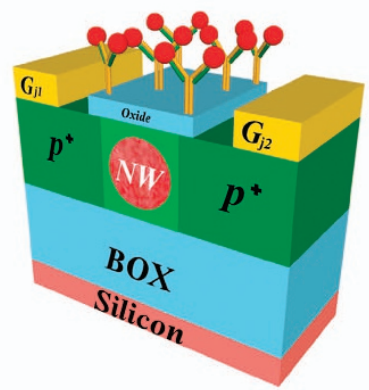

C

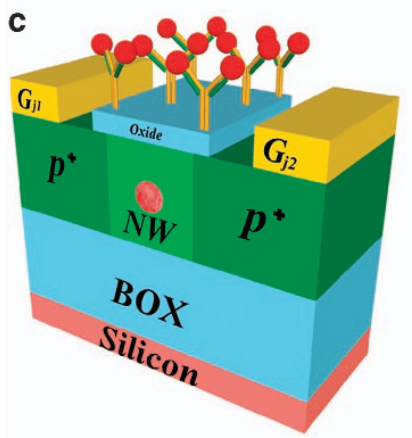

d

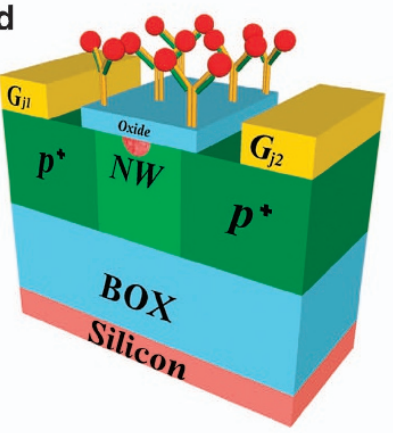

e

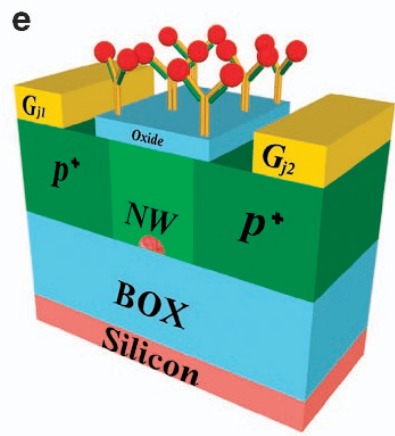

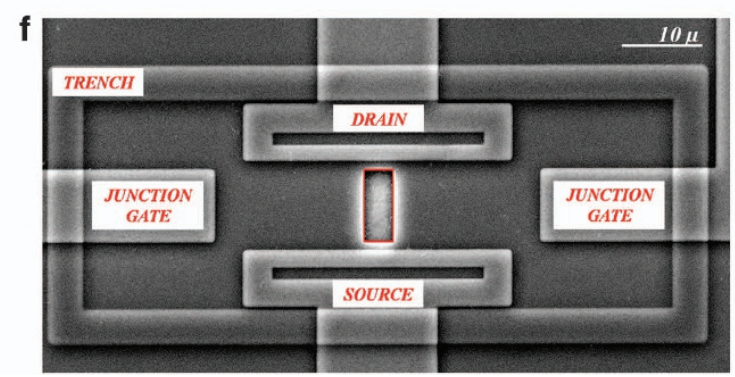

Figure 1 Schematic illustration of the EFN biosensor with antibody/analyte complexes bonded to the gate dielectric. (a) Note the $\mathrm{n}^{++}$and the $\mathrm{n}^{+}$implants for the source/drain and channel regions, respectively. The junction areas are $p^{+}$doped to allow efficient reverse biasing of the $p$ - $n$ junctions that electrostatically induce and control the width of the channel, using the electrical wiring shown in the inset. (b-e) Demonstrate the electrostatic control of the size, shape and location of the conducting channel. (b) Wide channel; (c) Reverse-bias forming a narrow channel; (d) Biasing of the top reference electrode and the backgate move the channel close to the biological event or (e) away from the noise centers at the $\mathrm{Si}_{/} \mathrm{SiO}_{2}$ interface. (f) $\mathrm{Scanning}$ electron microscope top image of the EFN device. The red rectangle in the middle of the device is the opening of the passivation layer where the modified gate dielectric is exposed to the analytes.

The EFN biosensor resembles the silicon-on-insulator (SOI) $\mathrm{G}^{4}$-FET developed in $2002^{16-18}$ and is presented schematically in Figure 1a. The biosensor is composed of an accumulation-type transistor with four, independent gates: a backgate $V_{\mathrm{Gb}}$, a frontgate/reference electrode $\left(V_{\mathrm{REF}}\right)$ and two additional lateral junction gates $\left(V_{\mathrm{Gj} 1}\right.$ and $V_{\mathrm{Gj} 2}$ ) located on both sides of the conducting channel. The nanoscale channel is induced and controlled by depletion regions formed around it by the four surrounding gates. In this way, the size, shape and location of the channel in the bulk SOI are determined. Figures $1 \mathrm{~b}$ and $\mathrm{c}$ illustrate an EFN biosensor with voltages $V_{\mathrm{Gj} 1}=V_{\mathrm{Gj} 2}$ that provide wide and narrow channels (nanowire dimension), respectively; the channel vertical position is controlled by $V_{\mathrm{Gb}}$ and $V_{\mathrm{REF}}$ as demonstrated in Figures $1 \mathrm{~d}$ and e. Figure 1f shows a scanning electron microscope top image of the EFN.

The EFN is conceptually different from both the conventional silicon nanowire (SiNW) and the double-gate nanowire. A SiNW is a three-dimensional structure in which the confinement is chemical (compositional), that is, it is due to a material (for example, air or $\mathrm{SiO}_{2}$ ) with different properties surrounding the wire. The confinement potential of the double-gate nanowire is chemical on the $\mathrm{SiO}_{2}$ side and electrostatic on the silicon side (this is an ordinary confinement potential of an inverted metal-oxide-silicon capacitor). On the contrary, in the EFN device, the confinement is fully electrostatic; that is, it is due to the external applied bias rather than chemistry. This fact implies that the interplay between sensing, charge carrier distribution and the confinement potential is inherently different in comparison to a SiNW or a double-gate nanowire (see the Supplementary Information for device simulations of the EFN parabolic potential vs the step-like potential in the SiNW and the double-gate nanowire). Additionally, degradation in the performance of SiNW-based devices due to metal catalysts and non-uniform dopant distribution ${ }^{19-22}$ does not exist in EFN biosensors because EFNs are electrostatically shaped inside singlecrystalline silicon. 
The EFN configuration allows the channel to be sufficiently removed from the $\mathrm{Si} / \mathrm{SiO}_{2}$ interface, ${ }^{23}$ which is the dominant noise source in SiNWs ${ }^{24-29}$ as well as in inversion-type planar devices ${ }^{30}$ (and in the double-gate nanowire, where the conduction is due to the formation of an inversion layer at the silicon $/ \mathrm{SiO}_{2}$ interface). It was recently demonstrated that the low-frequency noise originating from charge trapping/detrapping at the $\mathrm{Si} / \mathrm{SiO}_{2}$ interface is the prevailing noise source in wet environments, as the noise-level dependency on ion concentration and $\mathrm{pH}$ level is negligible. ${ }^{31-33}$ This EFN degree of freedom improves the signal-to-noise ratio, which is crucial for biosensing applications because the signal-to-noise ratio level will determine the sensitivity limit of the biosensor. ${ }^{34}$ Moreover, the ability to control the vertical position of the channel compromises between the need for maximum proximity of the biological events to the conducting channel on the one hand, and the need for high signal-tonoise ratio on the other. Figures $1 \mathrm{~d}$ and e illustrate the control of the vertical location of the EFN nanowire using solely the appropriate $V_{\mathrm{Gb}}$ and $V_{\mathrm{REF}}$. In Figure $1 \mathrm{~d}$, the channel is closer to the biological reaction and the noise centers (trap states that exchange electrons with the conducting channel) at the $\mathrm{Si} / \mathrm{SiO}_{2}$ interface, whereas in Figure 1e, the channel is further removed from the reacting surface and from the $\mathrm{Si} / \mathrm{SiO}_{2}$ interface.

As the confinement potential in the EFN is controlled electrostatically, the channel position can be changed in real time, and in this manner, a further increase in device sensitivity can be achieved (as examined in the Discussion section below). To summarize, the EFN allows post-fabrication determination of the channel size and shape, as well as the channel lateral and z-locations. In contrast, these parameters are predetermined and fixed in both SiNW and doublegate nanowires. We note that one of the advantages in biological detection using NWs is attributed to their large surface-to-volume ratio. $^{3-8}$ We demonstrate below that the sensitive sensing with the EFN biosensor is governed by the downscaling of the sensor active area to dimensions similar to that of the analytes. It should be noted that the EFN-enhanced sensitivity is not due to additional biasing as was argued for the dual-gated SiNW. ${ }^{35}$ An enhanced sensitivity was achieved with the dual-gated SiNW, once the shift in backgate threshold voltage was recorded (due to the ratio between the top dielectric and the buried dielectric). In the present case, only the shift in the front threshold voltage is measured, and hence the enhanced sensitivity is not due to the presence of additional gates.

\section{EXPERIMENTAL PROCEDURES}

\section{Silicon fabrication}

Silicon-on-insulator wafers measuring $6^{\prime \prime}$ in diameter were used (SOITEC, Bernin, France). The thickness of the SOI layer was $260 \mathrm{~nm}$, with boron doping of $1.6 \times 10^{14} \mathrm{~cm}^{-3}$ (resistivity of $13-22 \Omega \mathrm{cm}$ ). The thickness of the buried oxide was $1 \mu \mathrm{m}$. The fabrication of the devices was as follows: the devices were isolated from one another using isolation trenches. Successive implants for the channel region, the source/drain regions and junction regions were performed. The $\mathrm{SiO}_{2}$ gate dielectric of $50 \AA$ in thickness was grown via low-pressure chemical vapor deposition (LPCVD). The channel doping (As) was in the range of $1.6 \times 10^{17} \mathrm{~cm}^{-3}$. The EFN biosensor is partially depleted for the given channel doping and SOI thickness. The doping (As) at the source and drain regions was in the range of $5 \times 10^{19} \mathrm{~cm}^{-3}$, and the junction doping (B) was in the range of $5 \times 10^{19} \mathrm{~cm}^{-3}$. The source/drain and channel implants ensure an n-type accumulation device. The implant steps were followed by $100 \mathrm{~nm} \mathrm{SiO} 2$ plasma-enhanced chemical vapor deposition (PECVD) for the interlayer dielectric and opening of the contacts. Ti/Al/TiN was sputtered and patterned for interconnections followed by a $4500 \AA$ passivation layer of plasmaenhanced chemical vapor deposition nitride. The last step of the process was the opening of the passivation above the EFN biosensor-active region.
This step was performed with a dry etch followed by a final wet etch. Owing to the EFN geometry, two channel lengths need to be defined. The full channel length $(L)$ is defined as the lithographic length between the source and drain, and the effective length $\left(L_{\text {eff }}\right)$ is defined as the length of the junction gate (Figure 1a). All devices had a full channel length of $10 \mu \mathrm{m}$ and an effective length of $7 \mu \mathrm{m}$. The channel width $(W)$ for all devices was $400 \mathrm{~nm}$. See the Supplementary Information for schematic illustrations of the silicon processing.

Chemical modifications. Prior to modification, the devices were rinsed with EtOH (100\%), dried using $\mathrm{N}_{2}$ and activated with $\mathrm{UV} / \mathrm{O}_{3}$ (UVOCS T10X10, Montgomeryville, PA, USA) for $30 \mathrm{~min}$. Immediately after activation, the devices were dipped for $10 \mathrm{~min}$ in 1\% APTMS (3-aminopropyltrimethoxysilane, Sigma, Rehovot, Israel), in EtOH/DI (95:5) water solution, rinsed and sonicated for $10 \mathrm{~min}$ in $100 \% \mathrm{EtOH}$, dried in $\mathrm{N}_{2}$ and annealed for $10 \mathrm{~min}$ at $110^{\circ} \mathrm{C}$. The binding of either antibodies (anti-troponin and anti-PSA) to the APTMS was performed using a single-step preparation by dipping the APTMS-activated device for $2 \mathrm{~h}$ in $0.1 \times$ MES buffer $\mathrm{pH} 4.7$ supplemented with $5 \mathrm{~mm}$ 1-ethyl-3-(3-dimethylaminopropyl) carbodiimide (Pierce, Rockford, IL, USA) and $1 \mathrm{mg} \mathrm{ml}^{-1}$ polyclonal rabbit anti-troponin or $1 \mathrm{mg} \mathrm{ml}^{-1}$ polyclonal rabbit anti-PSA for the anti-troponin and anti-PSA devices, respectively (Abcam, Cambridge, MA, USA). The dies were washed twice with $0.1 \times$ MES buffer $\mathrm{pH} 4.7$, then once in phosphate buffer $\mathrm{pH}$ 6. The devices were measured immediately or stored under $\mathrm{N}_{2}$ until use.

Electrical connections. Electrical measurements of current vs applied reference-electrode voltage $\left(I_{\mathrm{DS}}-V_{\mathrm{REF}}\right)$ and current vs time $\left(I_{\mathrm{DS}}-t\right)$ of the EFN biosensor in a wet environment were performed with a custom-made flow cell. The measurements were performed with a homemade $\mathrm{Ag} / \mathrm{AgCl}$ reference electrode and $0.01 \times$ diluted commercial phosphate-buffered saline working solution with $10 \mathrm{~mm}$ sodium phosphate and $150 \mathrm{~mm} \mathrm{NaCl}$ at $\mathrm{pH} 7.4$ (Pierce). The electrical connectivity of the devices is illustrated in the inset of Figure 1a. Five voltages were applied to the devices: drain-source voltage $\left(V_{\mathrm{DS}}\right)$, voltage at the reference electrode $\left(V_{\mathrm{REF}}\right)$, back gate voltage $\left(V_{\mathrm{Gb}}\right)$ and two voltages at the junctions $\left(V_{\mathrm{Gj} 1}, V_{\mathrm{Gj} 2}\right)$. All measurements were performed with $V_{\mathrm{Gj}}=V_{\mathrm{Gj} 1}=$ $V_{\mathrm{Gi} 2}$. The $\mathrm{pH}$ sensitivity of EFN biosensors was measured with alternating injections of $0.01 \times$ phosphate-buffered saline solutions at $\mathrm{pH} 7$ and 9 . All the $I_{\mathrm{DS}}-t$ measurements were performed with $V_{\mathrm{DS}}=50 \mathrm{mV}$. The low value of $V_{\mathrm{DS}}$ was applied to guarantee the condition of $V_{\mathrm{DS}} \ll V_{\mathrm{Gj}}$. This condition provides a conducting channel with homogenous diameter throughout the length of the channel.

\section{RESULTS}

Figure $2 \mathrm{a}$ shows $I_{\mathrm{DS}}-V_{\mathrm{REF}}$ measurements of the EFN biosensor modified with cardiac troponin (anti-cTnI) for various junction gate voltages: $V_{\mathrm{Gj}}=0,-0.5,-1,-1.5$ and $-2 \mathrm{~V}$, in which the backgate voltage $\left(V_{\mathrm{Gb}}\right)$ was $-7 \mathrm{~V}$ for all devices. Note that $I_{\mathrm{DS}}$ decreases as $V_{\mathrm{Gj}}$ becomes more negative due to the presence of $\mathrm{p}-\mathrm{n}$ junctions on both sides of the channel formed by the boron and arsenic dopants. The p-n junctions control the effective width, $W_{\text {eff, of the conducting }}$ channel; reverse bias (negative $V_{\mathrm{Gj}}$ ) increases the depletion regions on both sides of the channel and hence reduces its width ( $I_{\mathrm{DS}}$ decrease), whereas forward bias (positive $V_{\mathrm{Gj}}$ ) reduces the depletion areas and consequently increases the channel width ( $I_{\mathrm{DS}}$ increase). The sensitivity of $I_{\mathrm{DS}}$ to variations in $V_{\mathrm{REF}}$ is characterized by the transistor gain $\left(g_{\mathrm{m}}=d I_{\mathrm{DS}} / d V_{\mathrm{REF}}\right)$, which is extracted from the $I_{\mathrm{DS}}-V_{\mathrm{REF}}$ measurements (Figure 2a). The shape of the $g_{\mathrm{m}}$ curve shows the expected single-peak signature for a device operation in the linear regime. The gain decreases as $V_{\mathrm{Gj}}$ becomes more negative (reversed bias), which is a direct consequence of the increase in the depletion regions at the junctions that reduce $I_{\mathrm{DS}}$ and $g_{\mathrm{m}}$. Finally, the shift in $g_{\mathrm{m}}$ towards higher values of $V_{\mathrm{REF}}$ as $V_{\mathrm{Gj}}$ is more negative, reflecting the shift in the front threshold voltage $\left(V_{\mathrm{Tf}}\right)$ due to a decrease in the front channel potential $\left(\psi_{\mathrm{sf}}\right){ }^{18}$ The value of $V_{\mathrm{REF}}$ (for a given $V_{\mathrm{Gj}}$ ) that provides the highest gain was selected for the $I_{\mathrm{DS}}-t$ measurements for 

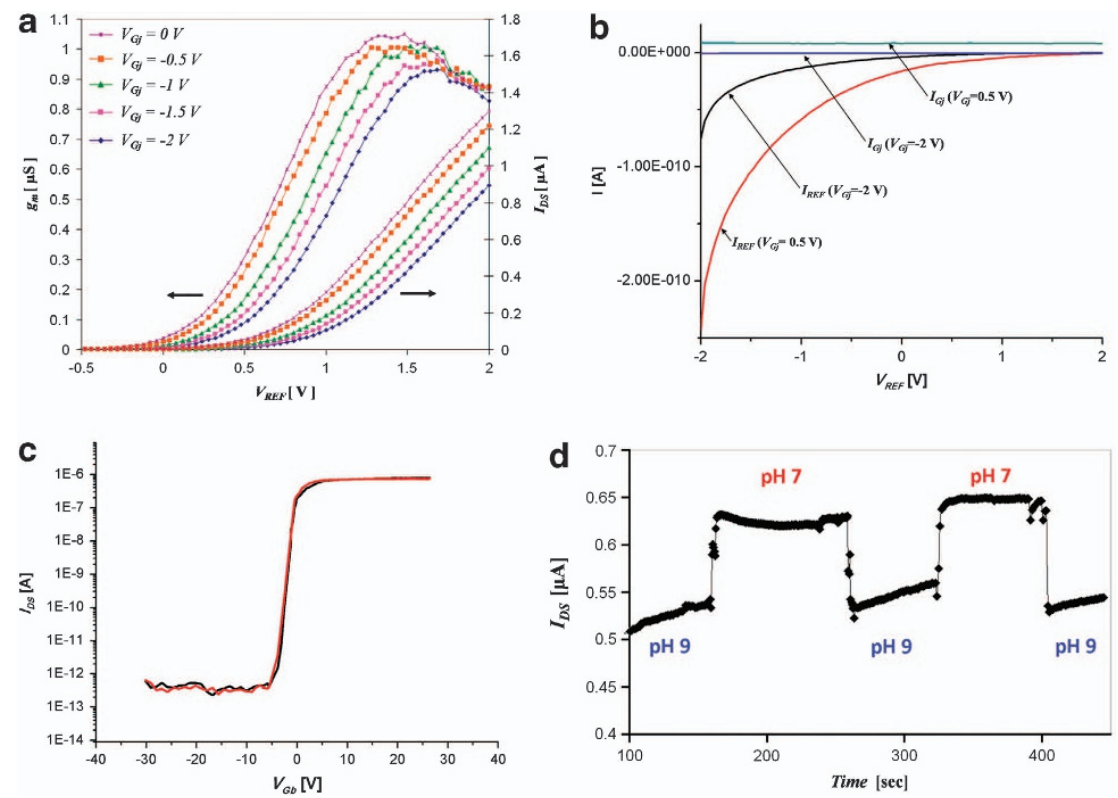

Figure 2 Electrical characterization of the EFN biosensor. (a) IDS Vs $V_{\text {REF }}$ and gain $\left(g_{\mathrm{m}}\right)$ curves of the EFN biosensor measured for different lateral gate voltages $\left(V_{G j}=0,-0.5,-1,-1.5\right.$ and $\left.-2 \mathrm{~V}\right)$. Note that as the junctions are more reverse biased, the $I_{D S}$ and the maximum value of $g_{m}$ are smaller because the effective width of the channel decreases. The measurements were performed in $0.01 \times$ phosphate-buffered saline buffer solution at $\mathrm{pH}$ 7. The channel is doped with $1.6 \times 10^{17} \mathrm{~cm}^{-3}$ of (As), the source/drain regions are doped with $5 \times 10^{19} \mathrm{~cm}^{-3}$ (As), and the junction areas are doped with $5 \times 10^{19} \mathrm{~cm}^{-3}$ (B). The lithographic width and length of the conducting channel are $400 \mathrm{~nm}$ and $7 \mu \mathrm{m}$, respectively. (b) Solution-related leakage currents in EFN. Leakage current at the reference electrode vs applied reference voltage for different biasing of junction gates. (c) Small hysteresis between forward and backward sweeps. (d) pH sensitivity of EFN biosensor. The modulation of $I_{\mathrm{DS}}$ due to alternating injections of $\mathrm{pH} 7$ and $\mathrm{pH} 9$ solutions is presented; the resulting $\mathrm{pH}$ sensitivity is $43 \mathrm{mV} \mathrm{dec}^{-1}$.

both the pH-sensitivity measurements and the measurements for analyte detection.

Solution-related leakage currents of an EFN modified with anticTnI are presented in Figure 2b. Note that the solution-junction gateleakage current $\left(I_{\mathrm{Gj}}\right)$ was measured for $V_{\mathrm{Gj}}=-2 \mathrm{~V}$ and $0.5 \mathrm{~V}$ for $V_{\mathrm{REF}}$ range of -2 to $2 \mathrm{~V}$. It is shown that $I_{\mathrm{Gj}}$ is not dependent upon $V_{\mathrm{REF}}$. As expected, the solution leakage current is greater when the $\mathrm{PN}$ junctions are forward-biased. However, the solution reference electrode leakage current $\left(I_{\mathrm{REF}}\right)$ exhibits strong dependence upon $V_{\mathrm{REF}}$, where negative $V_{\mathrm{REF}}$ implies an increase in $I_{\mathrm{REF}}$. Finally, $I_{\mathrm{REF}}$ is greater when the PN junctions are forward-biased, as expected. Drift currents $\left(I_{\mathrm{DS}}\right)$ were on the order of several $\mathrm{mV}$ for the initial $10 \mathrm{~h}$. Figure $2 \mathrm{c}$ presents the hysteresis between forward sweep and backward sweep. The small hysteresis between the two slopes is indicative of small, defect-induced charge trapping.

Figure $2 \mathrm{~d}$ shows the sensitivity to $\mathrm{pH}$ of the EFN biosensor modified with anti-cTnI for $V_{\mathrm{Gj}}=0 \mathrm{~V}\left(V_{\mathrm{DS}}=50 \mathrm{mV}, V_{\mathrm{REF}}=1.4 \mathrm{~V}\right.$, $\left.V_{\mathrm{Gb}}=-7 \mathrm{~V}\right)$. Alternating injections of $\mathrm{pH} 7$ and 9 solutions were performed, and as expected, higher $\mathrm{pH}$ entails smaller $I_{\mathrm{DS}}$ as the biologically modified gate dielectric is more negatively charged. The gain of the EFN biosensor was measured with a $V_{\mathrm{REF}}=-10 \mathrm{mV}$ calibration pulse, and in this manner, the shift in the front threshold voltage (due to the change in $\mathrm{pH}$ ) was extracted. The $\mathrm{pH}$ sensitivity of the device was calculated to be $43 \mathrm{mV} \mathrm{dec}^{-1}$, where the maximum theoretical value for the $\mathrm{pH}$ sensitivity of the $\mathrm{SiO}_{2}$ gate dielectric at this range of $\mathrm{pH}$ is $47-50 \mathrm{mV} \mathrm{dec}^{-1} .22$ A real-time, specific and labelfree detection of $10 \mathrm{pg} \mathrm{ml}^{-1}(\sim 340 \mathrm{fM}) \quad \mathrm{cTnI}$ is demonstrated in Figure 3a. The measurement was performed with narrow $W_{\text {eff }}$ $\left(V_{\mathrm{Gj}}=-2 \mathrm{~V}\right)$, and $V_{\mathrm{REF}}$ was selected to provide the highest $g_{\mathrm{m}}$ according to the $I_{\mathrm{DS}}-V_{\mathrm{REF}}$ plot. $V_{\mathrm{DS}}$ and $V_{\mathrm{Gb}}$ were set to $50 \mathrm{mV}$ and $-7 \mathrm{~V}$, respectively. The analyte injection occurred at $t=0$, where a sharp decrease in the current is recorded. This effect is in agreement with the isoelectric point of $\mathrm{cTnI}(\mathrm{pI}=5.2-5.4)$ that, in conjunction with the $\mathrm{pH}$ of the solution $(\mathrm{pH}=7.4)$, suggests that the troponin is negatively charged, and hence the current decrease is measured for the n-type channel. Non-specificity (with identical conditions) was also demonstrated for a device modified only with APTMS.

Figure $3 \mathrm{~b}$ presents the plots of normalized source-drain current vs time of six devices pre- and post-injection of $10 \mathrm{pg} \mathrm{ml}^{-1} \mathrm{cTnI}$. Each device was subjected to a different $V_{\mathrm{Gj}}$ to study the effect of the channel width upon threshold shift due to label-free and specific binding of cTnI. Four devices were operated with $V_{\mathrm{Gj}}=0,-0.5,-1$ and $-1.5 \mathrm{~V}$, and two devices with $V_{\mathrm{Gj}}=-2 \mathrm{~V}$. For each device, $V_{\mathrm{REF}}$ was selected to provide the largest $g_{\mathrm{m}}$ according to $I_{\mathrm{DS}}-V_{\mathrm{REF}}$ plots, and $V_{\mathrm{DS}}$ and $V_{\mathrm{Gb}}$ were set to $50 \mathrm{mV}$ and $-7 \mathrm{~V}$, respectively. The $g_{\mathrm{m}}$ for each plot was measured experimentally, and in conjunction with the shift in current, the shift in the front threshold voltage $\left(\Delta V_{\mathrm{Tf}}\right)$ due to the injection of cTnI was extracted. No modulation of current was recorded for $V_{\mathrm{Gj}}=0$ $\mathrm{V}$, whereas only a minute decrease in current of $2 \%$ was recorded for $V_{\mathrm{Gj}}=-0.5$ and $-1 \mathrm{~V}$, which provides $\Delta V_{\mathrm{Tf}}$ of $\sim 7 \mathrm{mV}$. However, a substantial decrease in current of $10 \%$ was demonstrated for $V_{\mathrm{Gj}}=-1.5 \mathrm{~V}\left(\Delta V_{\mathrm{Tf}}\right.$ of $\left.\sim 30 \mathrm{mV}\right)$, and a more pronounced decrease in current of $15 \%$ was demonstrated for the two devices subjected to $V_{\mathrm{Gj}}=-2 \mathrm{~V}$, which entails $\Delta V_{\mathrm{Tf}}$ of $\sim 73 \mathrm{mV}$ and $\Delta V_{\mathrm{Tf}}$ of $\sim 65 \mathrm{mV}$.

Figure $3 \mathrm{c}$ presents the EFN dose-response for $\mathrm{cTnI}$ concentrations of $10 \mathrm{pg} \mathrm{ml}^{-1}, 10 \mathrm{ng} \mathrm{ml}^{-1}$ and $10 \mu \mathrm{g} \mathrm{ml}^{-1}$ for $V_{\mathrm{Gj}}=-1.5 \mathrm{~V}$. Another EFN biosensor was modified with APTMS and anti-PSA, and the following voltages were applied: $V_{\mathrm{Gj}}=-2 \mathrm{~V}\left(W_{\text {eff }}=25 \mathrm{~nm}\right), V_{\mathrm{DS}}=50$ $\mathrm{mV}, \quad V_{\mathrm{REF}}=1.5 \mathrm{~V}$ and $V_{\mathrm{Gb}}=-7 \mathrm{~V}$. PSA at a concentration of $100 \mathrm{fg} \mathrm{ml}^{-1}(\sim 3 \mathrm{fM})$ was injected, and a real-time decrease in the current was evident. The decrease in current, with a corresponding, calculated threshold-potential shift of $\Delta V_{\mathrm{Tf}}=30 \mathrm{mV}$, is consistent 

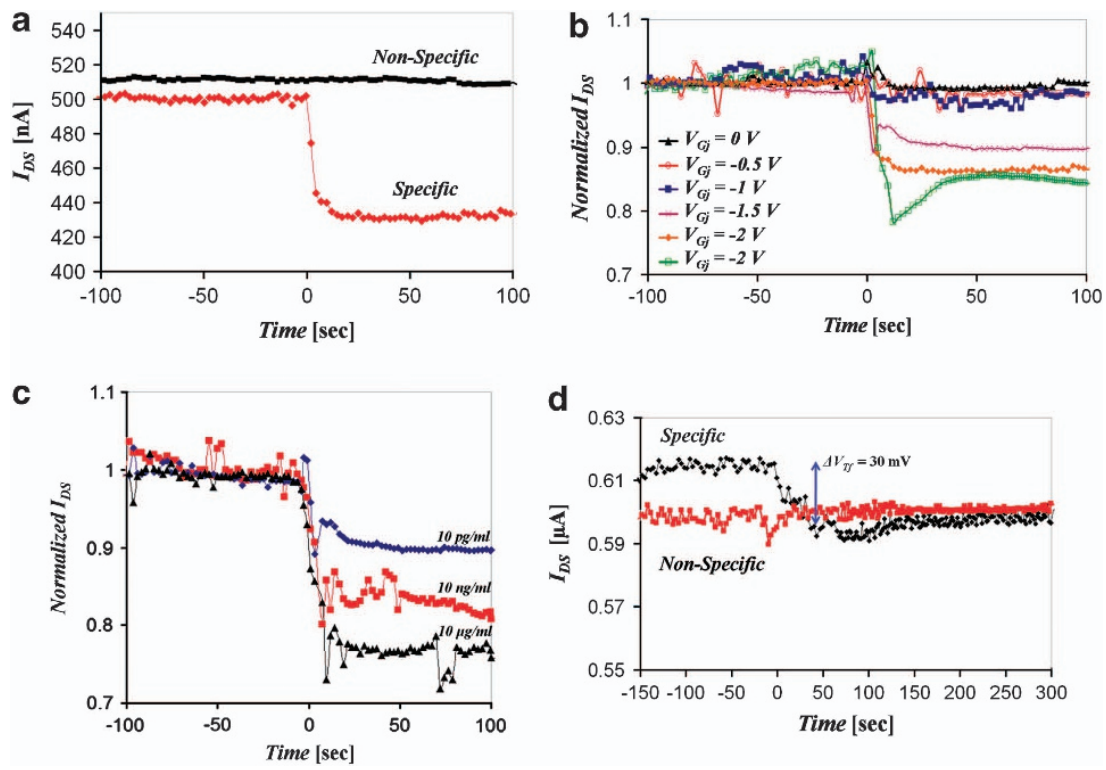

Figure 3 Specific, label-free and real-time detection with an EFN biosensor. The measurements were performed in a buffer solution of $0.01 \times$ phosphatebuffered saline at $\mathrm{pH}$ 7. (a) Response of $I_{\mathrm{DS}}$ to injection of $10 \mathrm{pg} \mathrm{ml}^{-1} \mathrm{cTnl}$ at $t=0 \mathrm{~s}$. The device was biased as follows: $V_{\mathrm{DS}}=50 \mathrm{mV}, V_{\mathrm{Gb}}=-7 \mathrm{~V}, V_{\mathrm{Gj}}=-2$ $V_{\mathrm{REF}}=1.6 \mathrm{~V}$. A decrease in $I_{\mathrm{DS}}$ of $15 \%$ is clearly observed following the cTnl binding. The decrease in current is expected for the given pl (5.2-5.4) of cTnI and the $\mathrm{pH}$ of the solution. Nonspecificity is also demonstrated because no change in current is apparent following injection of $10 \mathrm{pgml} \mathrm{m}^{-1}$ of $\mathrm{cTnl}$ to a device modified solely with APTMS (at $t=0 \mathrm{~s}$ ). (b) Normalized source-drain currents of six EFN biosensors subjected to $10 \mathrm{pg} \mathrm{ml}-1 \mathrm{cTnl}$ injections at $t=0 \mathrm{~s}$ and biased with $V_{\mathrm{DS}}$ and $V_{\mathrm{Gb}}$ of $50 \mathrm{mV}$ and $-7 \mathrm{~V}$, respectively. Each device was biased with a different $V_{\mathrm{Gj}}$ : $0 \mathrm{~V},-0.5 \mathrm{~V},-1 \mathrm{~V},-1.5 \mathrm{~V}$ and two devices with $-2 \mathrm{~V}$. For $V_{\mathrm{Gj}}=0 \mathrm{~V}$, no modulation in current is recorded, whereas for $V_{\mathrm{Gj}}$ of $-0.5 \mathrm{~V}$ and $-1 \mathrm{~V}$, only a minute current change is noticeable. Substantial current decreases of 10 and $15 \%$ are clearly observed for $V_{G j}=-1.5 \mathrm{~V}$ and for the two devices with $V_{G j}=-2 \mathrm{~V}$, respectively. (c) Normalized $I_{D S}$ vs time for the EFN dose response at $\mathrm{cTnl}$ concentrations of $10 \mathrm{pg} \mathrm{ml}^{-1}, 10 \mathrm{ng} / \mathrm{I}^{-1}, 10 \mu \mathrm{gml} \mathrm{l}^{-1}$. The applied voltages were $V_{\mathrm{DS}}=50 \mathrm{mV}, V_{\mathrm{Gb}}=-7 \mathrm{~V}, V_{\mathrm{Gj}}=-1.5$ $\left(W_{\text {eff }}=55 \mathrm{~nm}\right)$ and $V_{\mathrm{REF}}=1.6 \mathrm{~V}$. (d) Real-time, specific, and label-free detection of $3 \mathrm{fM} P S A$ was obtained for $V_{\mathrm{Gj}}=-2 \mathrm{~V}\left(W_{\text {eff }}=25 \mathrm{~nm}\right), V_{\mathrm{DS}}=50 \mathrm{mV}$, $V_{\mathrm{REF}}=1.5 \mathrm{~V}, V_{\mathrm{Gb}}=-7 \mathrm{~V}$. Note a shift of $30 \mathrm{mV}$ in threshold voltage. Nonspecificity is also demonstrated.

with the pI of PSA (5.8) and the pH of the solution (7.4) and suggests that the PSA is negatively charged (Figure $3 \mathrm{~d}$ ). Nonspecificity was verified by repeating the experiment with an identical device that was modified only with APTMS.

\section{DISCUSSION}

A three-dimensional device simulator (Synopsys TCAD Sentaurus, Mountain View, CA, USA) was used to calculate $W_{\text {eff }}$ as a function of $V_{\mathrm{Gj}} ; W_{\text {eff }}$ was estimated using the FWHM of the electron density of the conducting channel at the z-plane (vertical axis), where this density attains its maximum width (Figures $4 \mathrm{a}$ and $\mathrm{b}$ ). We note that $W_{\text {eff }}$ decreases from $190 \mathrm{~nm}$ for $V_{\mathrm{Gj}}=0 \mathrm{~V}$ to $25 \mathrm{~nm}$ for $V_{\mathrm{Gj}}=-2 \mathrm{~V}$ and that for $W_{\text {eff }}>80 \mathrm{~nm}\left(V_{\mathrm{Gj}}=-1 \mathrm{~V}\right)$, the current modulation upon binding of troponin is negligible. However, for $W_{\text {eff }}<$ than $80 \mathrm{~nm}$, the effect of the troponin binding is clearly observed. An even smaller EFN can be realized in the following way. Adequate thermal treatment will drive the boron dopants of the junction gates towards the center of the conducting channel and in this way form the PN junctions closer to the channel center. (See the Supplementary Information for simulations of $10 \mathrm{~nm}$ EFN).

The inverse correlation between $W_{\text {eff }}$ and the difference between the average potential of the depleted/accumulated SOI region pre- and post-analyte binding, $\Delta \psi_{\mathrm{SOI}}$, which is correlative to $\Delta V_{\mathrm{Tf}}$, was simulated and presented in Figure 4c. The EFN device was simulated with parameters identical to those of the measured devices (apart from the length of the device, which was set to $3 \mu \mathrm{m}$ to reduce computation time). A $1000 \mathrm{~nm}^{3} \mathrm{SiO}_{2}$ cube having a fixed charge of $10^{19} \mathrm{~cm}^{-3}$ was used to simulate the presence of a single-charged protein analyte, and $V_{\mathrm{Gj}}$ was changed from 0 to $-1.8 \mathrm{~V}$ in steps of $0.2 \mathrm{~V}$. Two simulations were performed for each $V_{\mathrm{Gj}}$, one with the localized charge in the channel center and the other one without any charge, and the average potential in the SOI region $\left(\psi_{\text {SOI }}\right)$ was extracted for each simulation. The difference in $\psi_{\text {SOI }}$ with and without charge was calculated for each value of $V_{\mathrm{Gj}}$. When no voltage is applied at the junctions $\left(V_{\mathrm{Gj}}=0 \mathrm{~V}\right.$ and $\left.W_{\text {eff }}=190 \mathrm{~nm}\right)$, the shift in $\psi_{\text {SOI }}$ is $2 \mathrm{mV}\left(W_{\text {eff }}=190 \mathrm{~nm}\right)$, whereas for $V_{\mathrm{Gj}}=-1.8 \mathrm{~V}\left(W_{\text {eff }}=37 \mathrm{~nm}\right)$, the shift is $\Delta \psi_{\mathrm{SOI}}=20 \mathrm{mV}$. Note that, as expected, a decrease in $W_{\text {eff }}$ entails a greater shift in $\psi_{\text {SOI }}$ (See the Supplementary Information).

Further modeling was used to extract the analyte charge density above the nanowire. Analytical modeling of the threshold voltage of a $\mathrm{G}^{4}$ transistor was recently reported. ${ }^{16}$ When the metal gate of the $\mathrm{G}^{4}$ transistor is removed and the gate dielectric is exposed to an electrolyte and a reference electrode, the metal work function $\left(\phi_{\mathrm{m}}\right)$ is replaced in the following manner ${ }^{36}: \phi_{\mathrm{m}} / q=E_{\mathrm{ref}}-\psi_{0}+\chi_{\mathrm{mol}}$, where $E_{\text {ref }}$ is the reference electrode potential relative to vacuum, $\psi_{0}$ is the double layer potential at the solution-oxide interface and $\chi_{\mathrm{mol}}$ is the dipole potential of the solution. For an EFN biosensor, we assume that the organic layers (APTMS, anti-cTnI and cTnI) can be modeled as a system of serially connected capacitors. Finally, we assume that the potential drops across the ATPMS layer, the anti-cTnI layer and $\psi_{0}$ did not change following the binding event. It was previously demonstrated - that using the above assumptions, the shift in front threshold voltage $\left(\Delta V_{\mathrm{Tf}}\right)$ is ${ }^{37}$ :

$$
\Delta V_{\mathrm{Tf}}=-\frac{Q_{\mathrm{cTnI}}}{C_{\mathrm{cTnI}}}=-\frac{Q_{\mathrm{cTnI}}^{*} \cdot t_{\mathrm{cTnI}}^{2}}{\varepsilon_{\mathrm{cTnI}}}
$$

where $Q_{\mathrm{cTn} I}, Q^{*}{ }_{\mathrm{cTnI}}, C_{\mathrm{cTnI}}, \varepsilon_{\mathrm{cTnI}}$ and $t_{\mathrm{cTnI}}$ are the overall charge, the charge density per unit volume (that is, the troponin charge density 

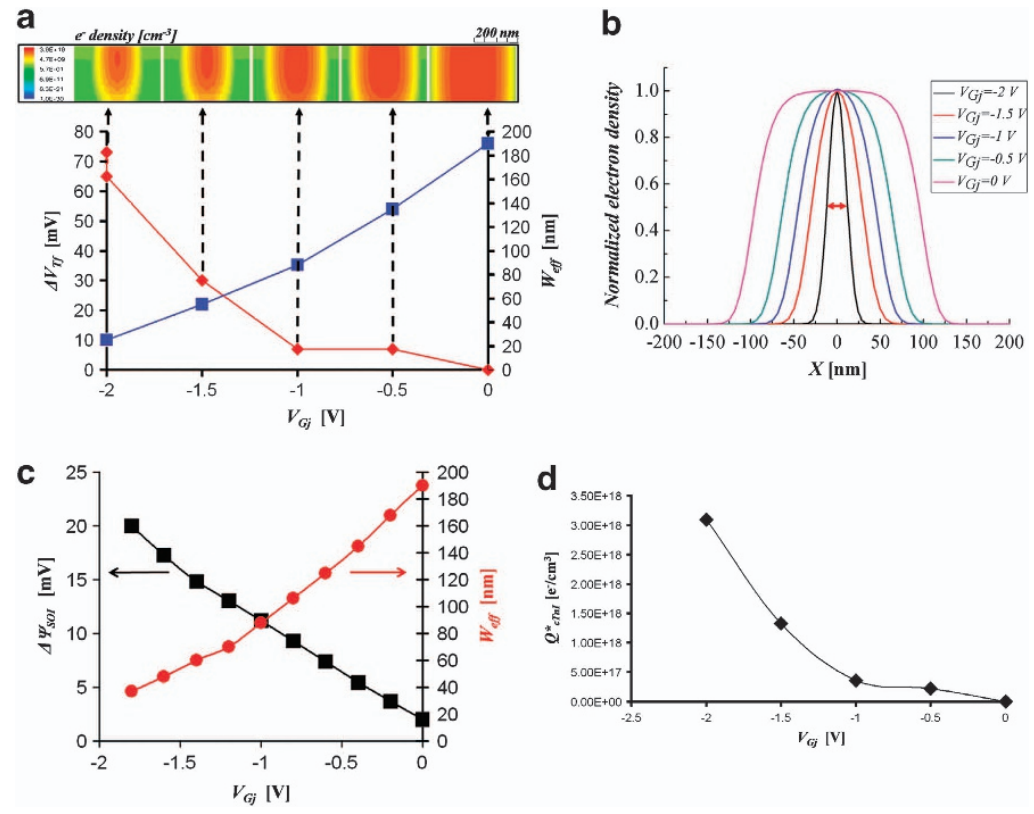

Figure 4 Simulations and modeling of the EFN biosensor. (a) The variation of the front threshold voltage, $\Delta V_{\text {Tf, }}$ and the calculated effective channel width, $W_{\text {eff }}$ (right axis), as a function of $V_{G j}$. The simulated cross-section of the electron density in the channel center at the middle of the channel $(x=L / 2)$ is shown (top) for each value of $V_{\mathrm{Gj}}$. Note the large increase in $\Delta V_{\mathrm{Tf}}$ with the formation of the nanoscale channel (smaller $W_{\text {eff }}$ ). A significant change in $\Delta V_{\mathrm{Tf}}$ is observed when $W_{\text {eff }}<80 \mathrm{~nm}$, and the induced channel is approaching the top surface, as is schematically shown above in Figure $1 \mathrm{~d}$. (b) The simulated profile of the normalized electron density in the middle of the channel $(x=L / 2)$ for each value of $V_{G j}$; a channel width of $25 \mathrm{~nm}$ at FWHM is obtained for $V_{G j}=-2$ V. (c) The variation of the average potential in the SOI region, $\Delta \psi_{\text {sol }}$, and $W_{\text {eff }}$ (red line, right axis) calculated using the Synopsys TCAD Sentaurus simulator for different $V_{\mathrm{Gj}}$. The simulated device includes only the inorganic components of the EFN biosensor, and the length of the device is set to $3 \mu \mathrm{m}$ rather than $7 \mu \mathrm{m}$. The change $\Delta \psi_{\mathrm{sol}}$ is calculated as the difference between a device with and without a fixed charge (on top of the gate dielectric) of $10^{19} \mathrm{~cm}^{-3}$ representing the analyte (see main text). The simulations support our observation that larger $V_{\mathrm{Gj}}$ (and consequently smaller $W_{\text {eff }}$ ) implies greater $\Delta \psi_{\mathrm{sol}}$ and enhanced detection sensitivity. (d) Calculated charge density $\left(Q^{*}{ }_{\mathrm{C} T n I}\right)$ as a function of $V_{\mathrm{Gj}}$ (this is the troponin charge density directly above the conducting nanowire). Note that as the gate voltage $V_{G j}$ is decreased from 0 to $-2 \mathrm{~V}$ and the corresponding channel width and vertical location change, the charge density of the cTnl layer over the conducting nanowire increases by one order of magnitude. This effect suggests that as $W_{\text {eff }}$ decreases, there is a probability of obtaining an analyte distribution that provides a greater effective charge density (the charge density just above the conducting nanowire), and hence, an enhanced device sensitivity.

directly above the conducting nanowire), the capacitance, the permittivity and the thickness of the cTnI layer, respectively (the Debye screening is neglected because the measurements were performed with $0.01 \times$ phosphate-buffered saline, which ensures a Debye length of $7 \mathrm{~nm}$ ). We assume a sparse cTnI layer (as full coverage of the APTMS layer and antibodies is not anticipated) with $\varepsilon_{\mathrm{cTnI}}=80$ and $t_{\mathrm{cTnI}}=10 \mathrm{~nm}$. The value of $Q^{*}{ }_{\mathrm{cTnI}}$ is calculated for each $V_{\mathrm{Gj}}$ and presented in Figure $4 \mathrm{~d}$. Note that as $W_{\text {eff }}$ decreases (or as $V_{\mathrm{Gj}}$ is more reverse-biased), there is a probability of obtaining an analyte distribution that provides greater charge density above the induced nanowire and consequently an enhanced device sensitivity. Finally, considering a typical dimension of $10 \mathrm{~nm}$ of a single cTnI molecule and $30 \%$ surface coverage of the cTnI layer, then the charge of a single cTnI molecule is $5 \mathrm{e}^{-}$, which is consistent with the theoretical calculations based on the typical Henderson-Hasselbalch equation (using software such as SEQMOL or PyMOL). Finally, the EFN detection sensitivity can be even further enhanced if a 'lateral voltage scan' mode is applied in the following manner. The two sidegate voltages $V_{\mathrm{Gj}} \mathrm{s}$ are continuously adjusted in such a way that the nanowire sweeps the channel from one $\mathrm{p}^{+}$region to the other (See the Supplementary Information and Supplementary Movie 1). In this way, the source-drain current is modified each time the EFN passes in close proximity to an analyte. This mode is similar to sensing using a NW array.

We have presented an electrostatically formed biosensor possessing a promising immunodetection performance in terms of real-time, specific and label-free detection with $\mathrm{fM}$ sensitivity. The conducting nanowire of the EFN biosensor is electrostatically defined post fabrication, which permits standard, CMOS-compatible silicon processing with extremely relaxed design rules. It was demonstrated that the electrostatically defined shape and position of the nanowire govern the detection sensitivity, that is, smaller the width of the nanowire, greater is the shift in the EFN threshold voltage. The EFN competitive sensing performance yields an inexpensive, disposable and ultrasensitive platform for various sensing applications.

\section{CONFLICT OF INTEREST}

The authors declare no conflict of interest.

1 Cui, Y., Wei, Q., Park, H. \& Lieber, C. M. Nanowire nanosensors for highly sensitive and selective detection of biological and chemical species. Science 293, 1289-1292 (2001).

2 Wang, W. U., Chen, C., Lin, K.-h., Fang, Y. \& Lieber, C. M. Label-free detection of small-molecule-protein interactions by using nanowire nanosensors. Proc. Natl. Acad. Sci. USA 102, 3208-3212 (2005).

3 Tang, T., Liu, X., Li, C., Lei, B., Zhang, D., Rouhanizadeh, M., Hsiai, T. \& Zhou, C. Complementary response of $\ln _{2} \mathrm{O}_{3}$ nanowires and carbon nanotubes to low-density lipoprotein chemical gating. Appl. Phys. Lett. 86, 1039031-1039033 (2005).

4 Zheng, G., Patolsky, F., Cui, Y., Wang, W. U. \& Lieber, C. M. Multiplexed electrical detection of cancer markers with nanowire sensor arrays. Nat. Biotechnol. 23, 1294-1301 (2005).

5 Stern, E., Klemic, J. F., Routenberg, D. A., Wyrembak, P. N., Turner-Evans, D. B., Hamilton, A. D., LaVan, D. A., Fahmy, T. M. \& Reed, M. A. Label-free immunodetection with CMOS-compatible semiconducting nanowires. Nature 445, 519-522 (2007). 
6 Kim, A., Ah, C. S., Yu, H. Y., Yang, J. H., Baek, I. B., Ahn, C. G., Park, C. W., Jun, M. S. \& Lee, S. Ultrasensitive, label-free, and real-time immunodetection using silicon fieldeffect transistors. Appl. Phys. Lett. 91, 1039011-1039013 (2007).

7 Hahm, J. i. \& Lieber, C. M. Direct ultrasensitive electrical detection of DNA and DNA sequence variations using nanowire nanosensors. Nano Lett. 4, 51-54 (2004).

8 Patolsky, F., Zheng, G., Hayden, O., Lakadamyali, M., Zhuang, X. \& Lieber, C. M. Electrical detection of single viruses. Proc. Natl. Acad. Sci. USA 101, 14017-14022 (2004).

9 Vu, X. T., Stockmann, R., Wolfrum, B., Offenhäusser, A. \& Ingebrandt, S. Fabrication and application of a microfluidic-embedded silicon nanowire biosensor chip. Phys. Status Solidi A. 207, 850-857 (2010).

10 Hsiao, C.-Y., Lin, C.-H., Hung, C.-H., Su, C.-J., Lo, Y.-R., Lee, C.-C., Lin, H.-C., Ko, F.-H., Huang, T.-Y. \& Yang, Y.-S. Novel poly-silicon nanowire field effect transistor for biosensing application. Biosens. Bioelectron. 24, 1223-1229 (2009).

11 Tong, H. D., Chen, S., van der Wiel, W. G., Carlen, E. T. \& van den Berg, A. Novel top-down wafer-scale fabrication of single crystal silicon nanowires. Nano Lett. 9, 1015-1022 (2009).

12 Garnett, E. C., Tseng, Y.-C., Khanal, D. R., Wu, J., Bokor, J. \& Yang, P. Dopant profiling and surface analysis of silicon nanowires using capacitance-voltage measurements. Nat. Nanotechnol. 4, 311-314 (2009).

13 Vaurette, F., Nys, J. P., Deresmes, D., Grandidier, B. \& Stievenard, D. Resistivity and surface states density of $\mathrm{n}$ - and p-type silicon nanowires. J. Vac. Sci. Technol. B. 26, 945-948 (2008).

14 Park, J. T., Kim, J. Y. \& Islam, M. Extraction of doping concentration and interface state density in silicon nanowires. IEEE T. Nanotechnol. 10, 1004-1009 (2011).

15 Ahn, J.-H., Choi, S.-J., Han, J.-W., Park, T. J., Lee, S. Y. \& Choi, Y.-K. Double-gate nanowire field effect transistor for a biosensor. Nano Lett. 10, 2934-2938 (2010).

16 Akarvardar, K., Cristoloveanu, S. \& Gentil, P. Analytical modeling of the twodimensional potential distribution and threshold voltage of the SOI four-gate transistor. IEEE T. Electron. Dev. 53, 2569-2577 (2006).

17 Akarvardar, K., Cristoloveanu, S., Gentil, P., Schrimpf, R. D. \& Blalock, B. J. Depletionall-around operation of the SOI four-gate transistor. IEEE T. Electron. Dev. 54, 323-331 (2007).

18 Dufrene, B., Akarvardar, K., Cristoloveanu, S., Blalock, B. J., Gentil, P., Kolawa, E. \& Mojarradi, M. M. Investigation of the four-gate action in $\mathrm{G}^{4}$-FETs. IEEE T. Electron. Dev. 51, 1931-1935 (2004).

19 Koren, E., Elias, G., Boag, A., Hemesath, E. R., Lauhon, L. J. \& Rosenwaks, Y. Direct measurement of individual deep traps in single silicon nanowires. Nano Lett. 11, 2499-2502 (2011)

20 Koren, E., Hyun, J. K., Givan, U., Hemesath, E. R., Lauhon, L. J. \& Rosenwaks, Y. Obtaining uniform dopant distributions in VLS-grown Si nanowires. Nano Lett. 11, 183-187 (2011).

21 Schlitz, R. A., Perea, D. E., Lensch-Falk, J. L., Hemesath, E. R. \& Lauhon, L. J. Correlating dopant distributions and electrical properties of boron-doped silicon nanowires. Appl. Phys. Lett. 95, 1621011-1621013 (2009).

22 Van Hal, R., Eijkel, J. \& Bergveld, P. A novel description of ISFET sensitivity with the buffer capacity and double-layer capacitance as key parameters. Sens. Actuators, B 24, 201-205 (1995).
23 Akarvardar, K., Dufrene, B. M., Cristoloveanu, S., Gentil, P., Blalock, B. J. \& Mojarradi, M. M. Low-frequency noise in SOI four-gate transistors. IEEE T. Electron. Dev. 53, 829-835 (2006)

24 Wei, C., Xiong, Y.-Z., Zhou, X., Singh, N., Rustagi, S. C., Lo, G. Q. \& Kwong, D.-L. Investigation of low-frequency noise in silicon nanowire MOSFETs in the subthreshold region. IEEE Electron. Dev. Lett. 30, 668-671 (2009).

25 Zhuge, J., Wang, R., Huang, R., Tian, Y., Zhang, L., Kim, D.-W., Park, D. \& Wang, Y. Investigation of low-frequency noise in silicon nanowire. IEEE Electron. Dev. Lett. 30, 57-60 (2009).

26 Reza, S., Bosman, G., Islam, M. S., Kamins, T. I., Sharma, S. \& Williams, R. S. Noise in silicon nanowires. IEEE T. Nanotechnol. 5, 523-529 (2006).

27 Rajan, N. K., Routenberg, D. A., Chen, J. \& Reed, M. A. Temperature dependence of $1 / f$ noise mechanisms in silicon nanowire biochemical field effect transistors. Appl. Phys. Lett. 97, 2435011-2435013 (2010).

28 Rajan, N., Routenberg, D., Chen, J. \& Reed, M. 1/f noise of silicon nanowire BioFETs. IEEE Electron. Dev. Lett. 31, 615-617 (2010).

29 Lee, J. W., Jang, D., Kim, G. T., Mouis, M. \& Ghibaudo, G. Analysis of charge sensitivity and low frequency noise limitation in silicon nanowire sensors. J. Appl. Phys. 107, 0445011-0445014 (2010).

30 Jakobson, C. \& Nemirovsky, Y. 1/f noise in ion sensitive field effect transistors from subthreshold to saturation. IEEE T. Electron. Dev. 46, 259-261 (1999).

31 Rajan, N., Routenberg, D. \& Reed, M. Optimal signal-to-noise ratio for silicon nanowire biochemical sensors. Appl. Phys. Lett. 98, 2641071-2641073 (2011).

32 Kim, S., Rim, T., Kim, K., Lee, U., Baek, E., Lee, H., Baek, C. K., Meyyappan, M., Deen, M. J. \& Lee, J. S. Silicon nanowire ion sensitive field effect transistor with integrated $\mathrm{Ag} / \mathrm{AgCl}$ electrode: $\mathrm{pH}$ sensing and noise characteristics. Analyst 136, 5012-5016 (2011).

33 Clément, N., Nishiguchi, K., Dufreche, J. F., Guerin, D., Fujiwara, A. \& Vuillaume, D. A silicon nanowire ion-sensitive field-effect transistor with elementary charge sensitivity. Appl. Phys. Lett. 98, 0141041-0141043 (2011).

34 Hassibi, A., Zahedi, S., Navid, R., Dutton, R. \& Lee, T. Biological shot-noise and quantum-limited signal-to-noise ratio in affinity-based biosensors. J. Appl. Phys. 97, 084701-084701 (2005).

35 Knopfmacher, O., Tarasov, A., Fu, W., Wipf, M., Niesen, B., Calame, M. \& Schonenberger, C. Nernst limit in dual-gated si-nanowire FET sensors. Nano Lett. 10, 2268-2274 (2010).

36 Bergveld, P. Thirty years of ISFETOLOGY: what happened in the past 30 years and what may happen in the next 30 years. Sens. Actuators B 88, 1-20 (2003).

37 Shalev, G., Rosenwaks, Y. \& Levy, I. The interplay between pH sensitivity and label-free protein detection in immunologically modified nano-scaled field-effect transistor. Biosens. Bioelectron. 31, 510-515 (2012).

\section{(c) (i) $(-)$ This work is licensed under a Creative Commons Attribution-NonCommercial-NoDerivs 3.0 Unported License. To view a copy of this license, visit http://creative commons.org/licenses/by-nc-nd/3.0/}

Supplementary Information accompanies the paper on the NPG Asia Materials website (http://www.nature.com/am) 